\title{
Syndrome de Melkersson-Rosenthal. À propos de deux observations
}

\author{
Melkersson-Rosenthal syndrome: two cases report
}

\section{RÉSUMÉ}

Le syndrome de Melkersson-Rosenthal (SMR) est une pathologie rare caractérisée dans sa forme complète par l'association d'un œdème récidivant de la face, d'une paralysie faciale périphérique et d'une langue plicaturée. L'étiologie et la pathogénie de ce syndrome restent encore inconnues mais plusieurs théories ont été proposées : immuno-allergiques, infectieuses (bactérienne ou virale) ou génétiques.

La symptomatologie est le plus souvent incomplète. La macrochéilie de Miescher est considérée par la plupart des auteurs comme une forme monosymptomatique de ce syndrome. Le diagnostic est basé sur l'histologie qui met en évidence des granulomes lympho-épithéloïdes, parfois giganto-cellulaires sans nécrose caséeuse. Le traitement n'est pas bien codifié : il est avant tout symptomatique, médical parfois chirurgical. Med Buccale Chir Buccale 2007; 13 : 143-151.

mots clés: syndrome de Melkersson-Rosenthal, diagnostic, traitement

médecine buccale chirurgie buccale

VOL. $13, N^{\circ} 3$ 2007

page 143

\section{SUMMARY}

The Melkersson-Rosenthal syndrome which is a rare pathology is characterised in its complet form by the association of the face's offensive oedema, a peripheric facial paralysis and a plicatured tongue. The etiology and the pathogenicity of this syndrome remain unknown, however certain immuno-allergic, infectious (bacteriel or viral) and genetic theories exist.

The granulomatous macrocheilitis of Miescher is considered by the most of authors as a monosymptomatical form of this syndrome.

The diagnosis essentially based on the histology which makes is evidence lymphoepitheliogranuloma without casous necrosis.

The treatment is not defined, but it is symptomatic: medical sometimes chirurgical. Med Buccale Chir Buccale 2007; 13 : 143-151.

key words: Melkersson-Rosenthal syndrome, diagnosis, treatment 
Le syndrome de Melkersson-Rosenthal (SMR) est une affection rare. C'est une granulomatose faciale touchant les deux sexes avec une triade de manifestations buccofaciales évocatrices, caractérisée par l'association concomitante d'un œdème labial asymétrique, d'une paralysie faciale homolatérale et d'une langue présentant des plicatures. L'étiopathogénie est inconnue. Le diagnostic positif est essentiellement clinique et le chirurgien dentiste peut jouer un rôle important dans le diagnostic précoce de cette maladie.

Cet article se propose de discuter, à partir de deux cas cliniques de SMR et, à l'appui des données de la littérature, les différentes possibilités diagnostiques et thérapeutiques devant les manifestations cliniques considérées comme caractéristiques du SMR.

médecine

buccale

chirurgie

buccale

VOL. $13, \mathrm{~N}^{\circ} 3$ 2007

page 144
L'interrogatoire révèle l'apparition d'une tuméfaction labiale en 2000 qui a été attribuée à une allergie alimentaire et qui a régressé spontanément, des paresthésies et une impression de paralysie faciale évoluant par épisodes.

Une nouvelle poussée de macrochéilie et d'œdème de la face est survenue deux années plus tard ; elle a été mise sur le compte d'un abcès dentaire. Cet épisode n'a régressé que partiellement. D'autres poussées se sont succédé sans facteurs déclenchants apparents. L'examen exobuccal montre une macrochéilie asymétrique, un œdème intéressant l'étage moyen de la face et la région latéro-mandibulaire droite (Fig. 1a et 1b). A la palpation, cette tuméfaction présente une consistance ferme et fibreuse. A l'examen endobuccal (Fig. 1c), on retrouve un aspect normal de la langue et un mauvais état buccodentaire (nombreuses caries den-

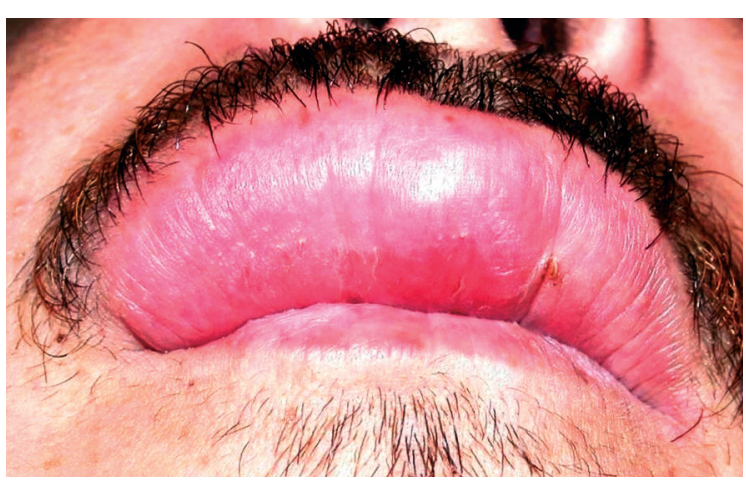

Figure 1b : Vue exobuccale. Macrochéilie asymétrique. Exobuccal view: asymmetrical macrochilia.

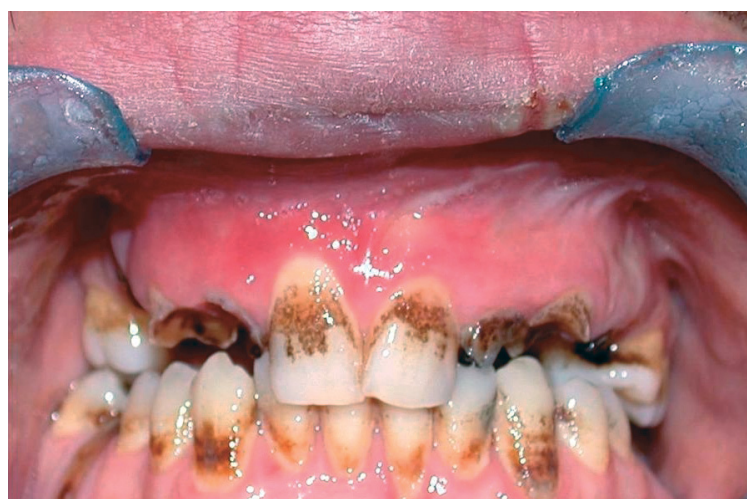

Figure 1c: Vue endobuccale. Gingivite avec changement de couleur coïncidant avec le côté de macrochéilie.

Intraoral view: gingivitis with change of color coinciding with the side of macrochilia. 
taires et plusieurs dents à l'état de racine). L'examen parodontal montre une gingivite avec une couleur différente du côté de la macrochéilie. Une thérapeutique dento-parodontale a été alors instaurée avec motivation et instructions pour l'hygiène bucco-dentaire, détartrage, extraction des dents non conservables, et traitement des caries. La biopsie gingivale a confirmé le diagnostic en mettant en évidence une gingivite granulomateuse avec une inflammation riche en plasmocytes et quelques granulomes épithélio-giganto-cellulaires sans nécrose caséeuse (Fig. 2).

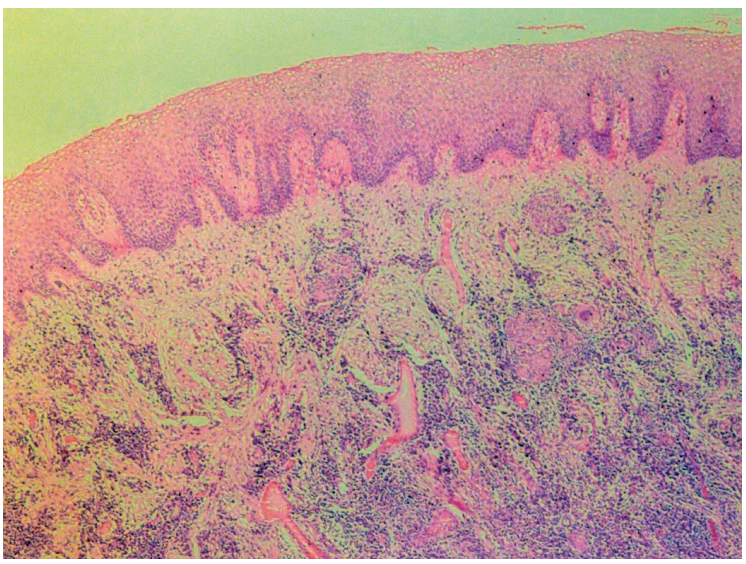

Figure 2 : Aspect histologique. Granulomes épithéliogiganto-cellulaires sans nécrose caséeuse. (Hématéineéosine, 40).

Histological aspect: epithelio-giganto-cellular granulomas without necrosis (Hematein-eosin, 40).

Une semaine après la fin des soins, il y a une disparition de la gingivite, une diminution spontanée de l'aspect fibreux de la granulomatose (Fig. 3) et

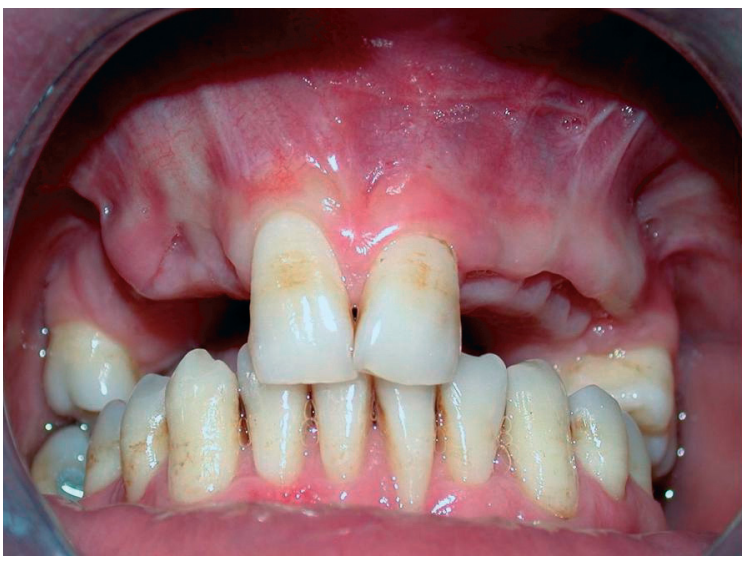

Figure 3 : Vue endobuccale. Gencive d'aspect sain. Intraoral view: gum of healthy aspect. de l'œdème. Deux semaines après la fin des soins dentaires, un traitement à base de corticoïdes a été entrepris à la dose de $1 \mathrm{mg} / \mathrm{kg} / \mathrm{j}$ d'équivalent de prednisone par le médecin interniste. Après 1 mois de traitement, la régression de l'œdème est encore plus nette (Fig. 4).

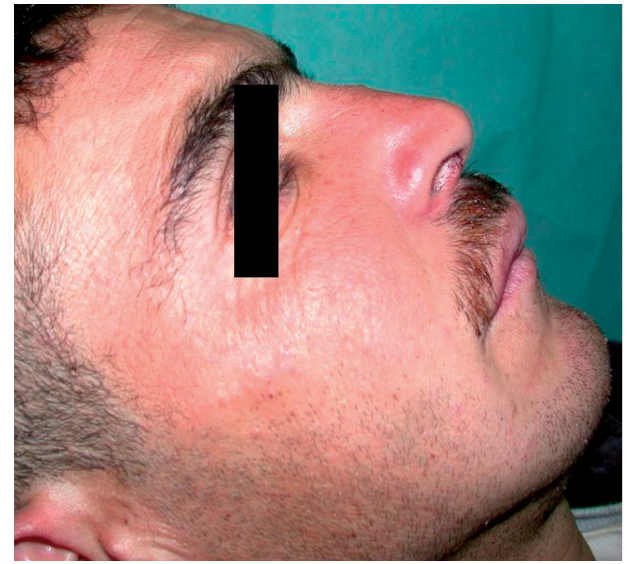

Figure 4 : Après 1 mois de traitement. Réduction significative de l'œdème.

To 1 month of treatment: significant reduction of the oedema.

\section{DEUXIÈME OBSERVATION}

médecine

buccale

chirurgie

buccale

VOL. $13, \mathrm{~N}^{\circ} 3$

2007

page 145
Un patient âgé de 40 ans, a consulté en novembre 2004 dans le service d'Odontologie chirurgicale pour une sensation d'engourdissement et une tuméfaction intéressant la région labiale et l'hémiface droites. Dans les antécédents médicaux, on note une paralysie faciale droite en 1998, une maladie de Crohn évoluant depuis 2000 mais stable sous traitement, un épisode de tuméfaction des lèvres en 2001 attribué à une infection d'origine dentaire mais il a régressé spontanément. Deux années plus tard, le patient a présenté une nouvelle poussée de macrochéilie et d'œdème facial sans facteurs favorisants apparents. L'examen exobuccal a révélé (Fig. 5a et 5b) un œdème labial supérieur et inférieur, une tuméfaction de l'hémiface droite, les tissus de recouvrement sont d'aspect normal. A la palpation, cette tuméfaction est élastique mais de consistance variable, indolore, non fluctuante et sans adénopathie associée. L'examen endobuccal montre une langue œdématiée (Fig. $5 \mathrm{c}$ ) et une muqueuse jugale lobulée (Fig. 6), prenant un aspect 


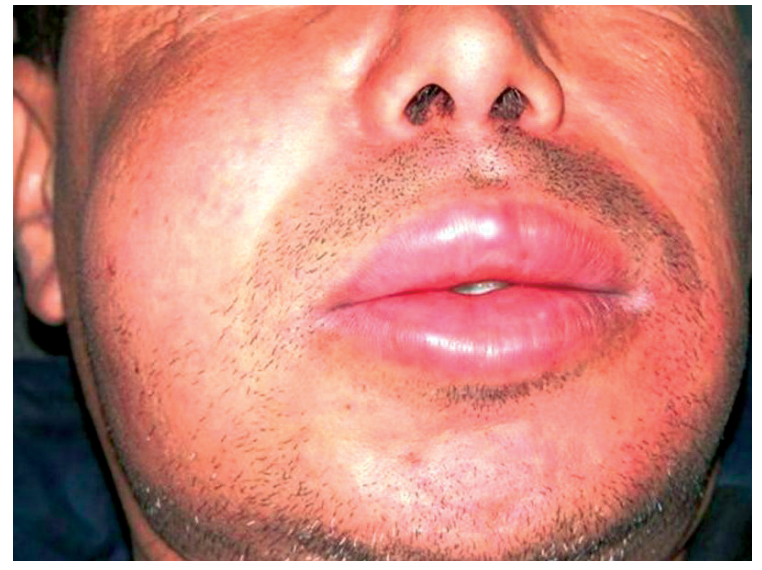

Figure 5a : Vue de face. CEdème de l'hémiface droite. Front view of the face: oedema of the right hemiface.

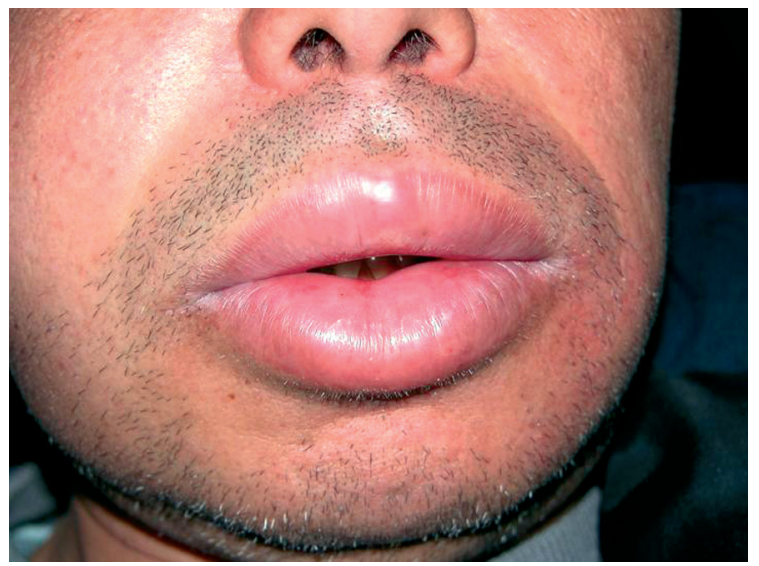

Figure 5b : Vue exobuccale. Macrochéilie labiale. Exobuccal view: labial macrochilia.

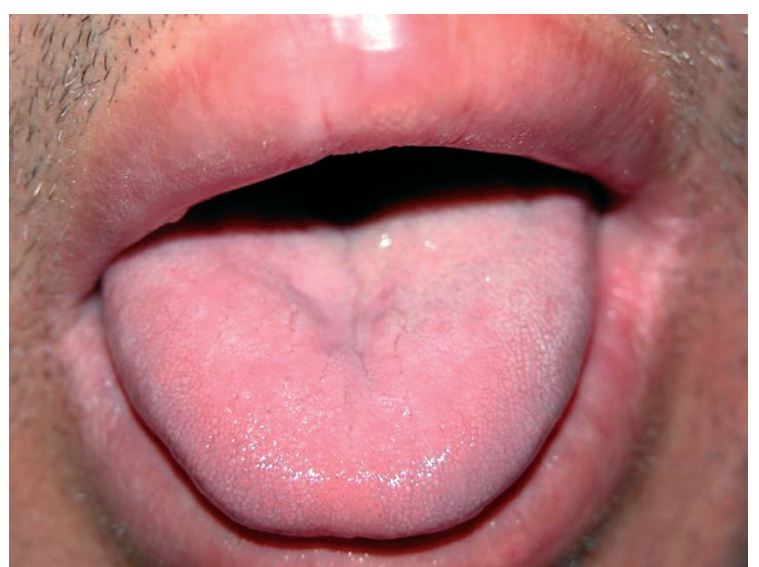

Figure 5c : Vue endobuccale. Langue tuméfiée. Intraoral view: tumefied tongue.

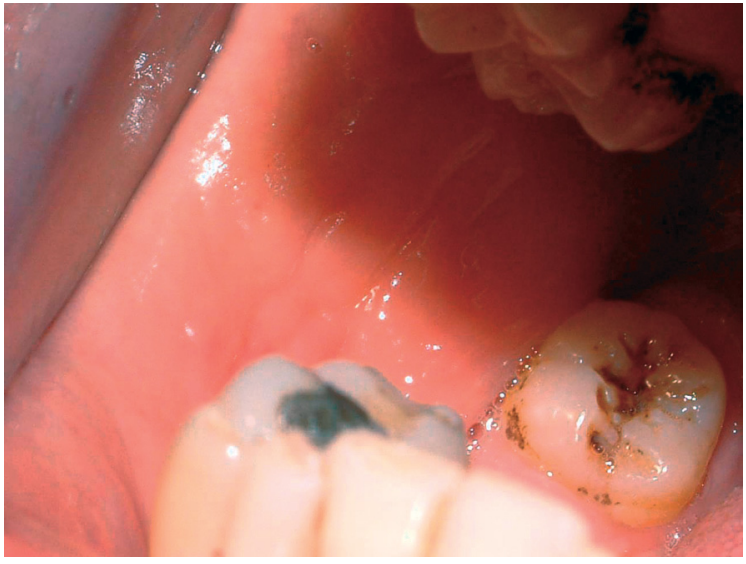

Figure 6 : Vue endobuccale. Aspect lobulé de la muqueuse jugale.

Intraoral view: lobule aspect of the mucosa jugal.

carrelé. L'examen dentaire décèle la présence de multiples caries dentaires.

Devant l'association œdème de la face et sensations d'engourdissement facial, le diagnostic de SMR a été évoqué. Une biopsie de la muqueuse jugale et labiale a été réalisée ; l'examen anatomopathologique a montré la présence de granulomes épithélio-giganto-cellulaires sans nécrose caséeuse (Fig. 7).

Le patient a été confié à un médecin interniste qui a retenu le diagnostic de SMR et qui a entrepris une corticothérapie (30 mg de prednisone/j) pendant 1 mois. L'évolution a été marquée par des périodes de rémission au cours desquelles l'œdème buccofacial régressait de manière significative (Fig. 8).

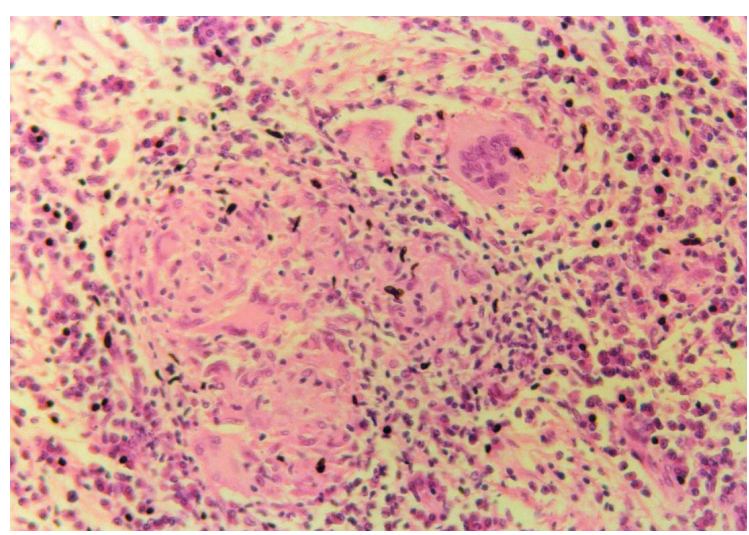

Figure 7 : Aspect histologique. Granulomes épithéliogiganto-cellulaires sans nécrose caséeuse (Hémateineeosine, 400).

Histological aspect: epithelio-giganto-cellular granulomas without necrosis (Hematein-eosin, 400). 


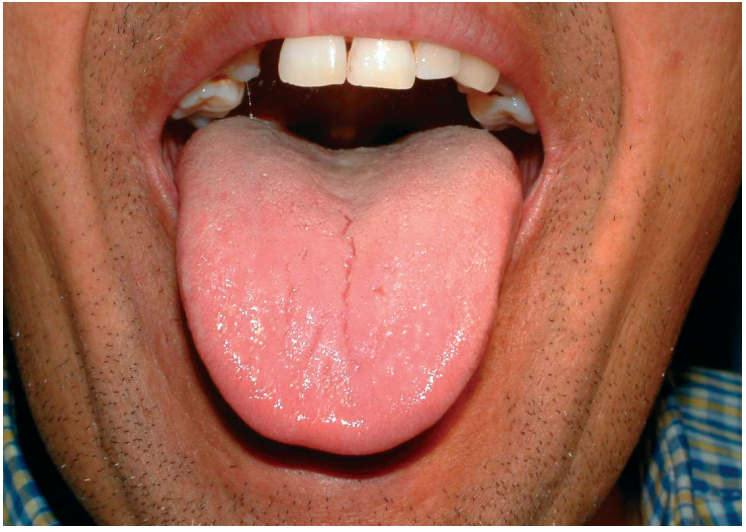

Figure 8 : Vue endobuccale. Aspect normal des lèvres et de la langue après le traitement.

Intraoral view: normal aspect of the lips and the tongue after the treatment.

\section{COMMENTAIRES}

En 1928, Melkersson décrit un œdème facial et une paralysie faciale chez une patiente âgée de 35 ans et suggère qu'il existe une relation entre les deux symptômes. Rosenthal rajoute, en 1931, un nouveau symptôme, la langue plicaturée. La triade sera désignée, en 1949, sous le nom de SMR [1].

Le SMR survient chez l'adulte jeune, le plus souvent après la fin de la $2^{\mathrm{e}}$ décennie, sans prédominance de sexe, ni de race [2]. Selon une étude réalisée sur 36 patients, Greenne et Rogers (1989) ont noté que l'âge moyen lors du diagnostic était de 39 ans et que l'âge moyen au début des symptômes de 33 ans ${ }^{[3]}$.

La pathogénie du SMR reste inconnue. Stosiek et coll. (1992) proposent une théorie inflammatoire : la présence d'antigènes circulants non spécifiques entraînerait des troubles vasomoteurs des petits vaisseaux de l'extrémité céphalique, donnant des œdèmes sous-cutanés et des gaines nerveuses ${ }^{[4]}$. D'autres mécanismes ont été évoqués : anomalies du drainage lymphatique, phénomènes allergiques (aliments, cosmétiques, certains composants des produits utilisés pour l'hygiène buccale), origine bactérienne ou virale (herpès, toxoplasmose, syphilis...), rôle des foyers infectieux dentaires et parodontaux [1,4]. Effectivement l'élimination des foyers infectieux d'origine dentaire souvent présents chez les por- teurs de SMR peut amener une amélioration parfois spectaculaire de l'œdème, ce qui semble plaider en faveur de la théorie infectieuse notamment bactérienne, mais il n'existe pas de preuves scientifiques réelles [2]. Le SMR pourrait représenter une forme particulière de sarcoïdose ou de maladie de Crohn. Actuellement, beaucoup d'auteurs considèrent qu'il existe une prédisposition constitutionnelle ou héréditaire.

Le diagnostic de SMR est essentiellement clinique. Meisel-Stosiek et Hornstein (1990) ont proposé une classification qui rend compte des manifestations cliniques très polymorphes de ce syndrome (Tab. 1). Un SMR typique comporte des symptômes du $1^{\mathrm{er}}, 2^{\mathrm{e}}$ et $3^{\mathrm{e}}$ ordres. C'est la connaissance parfaite de ces différents symptômes qui peut guider le praticien dans son diagnostic ${ }^{[5]}$. Cependant, un examen anatomopathologique des tissus œdématiés se révélera d'une aide précieuse pour l'orientation du diagnostic [5].

\section{Symptômes majeurs ou de $1^{\text {er }}$ ordre}

Il y en a deux : I'œdème buccofacial, avec son aspect histologique typique, et la paralysie faciale. L'œdème buccofacial est inaugural dans 33 à $42 \%$ des cas, souvent asymétrique. II est au début intermittent, puis permanent; il peut être exobuccal et intéresser les lèvres, les joues, les paupières ou bien il est endobuccal et toucher la gencive, la muqueuse jugale, la langue. L'œdème labial est présent dans $70 \%$ des cas de SMR; il intéresse essentiellement la lèvre supérieure et survient plutôt de façon brutale, sans véritables prodromes. Parfois les patients signalent l'existence de signes précurseurs tels que des névralgies faciales, des migraines, des maux de tête. Une sensation de tension ou de piqûre dans la lèvre a été aussi rapportée [1,2, 6]. Cliniquement, on note un épaississement de la lèvre qui donne parfois un aspect de "groin » de face ou de «museau de tapir » de profil. Progressivement, la lèvre s'éverse, exposant ainsi la partie muqueuse ; la lèvre peut atteindre deux à trois fois sa taille normale [2, 6, 7]. L'œdème est souvent asymétrique ${ }^{[6]}$, le côté le plus atteint correspond presque toujours à celui de la paralysie faciale ${ }^{[6]}$. La région œdématiée peut être légèrement cyanosée, pre- médecine

buccale

chirurgie

buccale

VOL. $13, \mathrm{~N}^{\circ} 3$ 2007

page 147 
médecine

buccale

chirurgie

buccale

VOL. $13, \mathrm{~N}^{\circ} 3$ 2007

page 148 nant alors une couleur rouge violacée, surtout dans les formes sévères. Mais dans bien des cas cependant, les téguments conservent une couleur normale. A la palpation, la lèvre a une consistance variable mais il n'y a jamais de fluctuation. On perçoit parfois des petits nodules qui correspondent à l'inflammation granulomateuse [7, 8]. L'épisode œdémateux est souvent de courte durée, tout au moins au début de la maladie. II persiste un jour ou deux, voire une semaine mais rarement au-delà. Lors du premier épisode, l'œdème disparaît sans laisser de traces, pour réapparaître de façon identique, le plus souvent à la même place, mais à intervalles irréguliers, allant de quelques semaines à quelques mois. Après plusieurs épisodes, l'œdème perdure et se résorbe lentement et partiellement, puis devient finalement permanent dans de nombreux cas $[1,5,8]$. Selon l'importance de l'œdème, on peut observer une incontinence labiale, une dysfonction labiale importante, des difficultés d'élocution ou d'alimentation, et un préjudice esthétique. Les lèvres présentent parfois des fissures et une coloration comme cela a été observé pour le 1er patient [2].

D'autres localisations œdémateuses céphaliques ont été rapportées, notamment l'atteinte des joues (macroparéite), du nez, des paupières (blépharite), du front (métopite) ou de toute la face (prosopite) [7, 9]. Ziemmer et coll. (1992) ont décrit les atteintes endobuccales qui peuvent siéger dans la muqueuse jugale (12\%), les gencives (19\%), le voile du palais (macroulie), la langue (17\%) [10]. La muqueuse buccale, et particulièrement la gencive, peut être le siège d'hypertrophie, d'inflammation et d'érosions douloureuses.

Le SMR s'inscrit dans le cadre des granulomatoses buccofaciales (orofacial granulomatosis). Une hypertrophie gingivale peut constituer la première manifestation d'une granulomatose $[2,10]$. Le mode d'évolution des lésions endobuccales est identique à celui des manifestations exobuccales du SMR [11], ce qui permet de faire le diagnostic différentiel avec la gingivite commune [9].

La paralysie faciale est de type périphérique. Elle apparaît après un ou plusieurs épisodes d'œdème ; elle est présente dans 33 à $47 \%$ des cas [11, 12]. Quelquefois, elle peut survenir des mois, voire des années avant l'œdème. Elle apparaît soit de façon brutale, très souvent constituée au cours de la nuit et constatée au réveil, soit de façon progressive en vingt quatre à quarante huit heures. Elle est en général unilatérale et exceptionnellement bilatérale ${ }^{[9,10,11]}$. Elle intéresse tous les muscles de la face, le territoire facial supérieur et inférieur étant touchés de façon identique. On peut cependant observer des formes incomplètes [11]. Le côté atteint par la paralysie correspond classiquement à celui touché par l'œdème [11,12]. Elle peut être accompagnée d'autres atteintes nerveuses se traduisant par une hyperacousie douloureuse, une agueusie intéressant les 2/3 antérieurs de la langue, une altération des sécrétions lacrymales, nasales et salivaires.

\section{Symptômes de $2^{\mathrm{e}}$ et de $3^{\mathrm{e}}$ ordres (Tab. 1)} La langue est plicaturée ou scrotale dans 20 à $60 \%$ des cas ${ }^{[5]}$. Elle peut apparaître vers l'âge de 4 ans, et pourrait parfois déjà être présente à la naissance. Elle serait transmise suivant un mode autosomique dominant irrégulier et semble en corrélation avec une langue géographique qui, le plus souvent, la précède dans le temps ${ }^{[1]}$. La langue plicaturée constitue donc un signe caractéristique mais sans signification diagnostique. Des signes neurovégétatifs sont assez fréquents : larmoiements ou yeux secs, hypersialorrhée ou asialie, dysgueusie, hyperacousie, troubles de la sudation ${ }^{[6]}$. Des migraines, des paresthésies et des troubles de l'humeur ont été aussi rapportés ${ }^{[4]}$. Une atteinte du nerf optique et des anomalies des vaisseaux rétiniens ont été observées. Sur le plan anatomopathologique, il peut exister deux types histologiques. Le type granulomateux caractérisé par la présence des granulomes épithélio-giganto-cellulaires sans nécrose caséeuse, qui ressemblent aux granulomes sarcoïdosiques dont ils se distinguent par la taille plus petite et le caractère non confluent [13]. Le type lympho-nodulaire plasmocytique qui se voit spécialement dans les tissus facilement œdémateux comme les paupières ${ }^{[2,8]}$.

La macrochéilie granulomateuse est un signe constant du SMR, mais on la retrouve également dans le tableau clinique de la sarcoïdose et de la maladie de Crohn. Le diagnostic différentiel avec ces deux affections est parfois difficile. Tatnal et 
coll. (1987) ont été les premiers à proposer une éventuelle relation entre la SMR et la maladie de Crohn, en se basant sur la similitude histologique existant dans l'œdème labial. Cependant, aucune manifestation caractéristique de cette affection (douleurs abdominales, diarrhées hémorragiques...) n'est rencontrée dans le SMR [14, 15,16]. Dans la sarcoïdose, le granulome est bien différent de celui du SMR. Cependant, la distinction peut s'avérer difficile lorsqu'il existe une inflammation importante [1, 11]. Ang et Jones (2002), dans une étude portant sur 7 patients atteints de SMR, ont trouvé une calcémie et un taux d'angiotensine convertase normaux, et une réactivité négative au test de Kveim ; ces résultats biologiques semblent bien confirmer que la SMR et la sarcoïdose sont deux entités nosologiques différentes ${ }^{[9]}$. Devant un œdème buccofacial, le chirurgien dentiste pense d'emblée à une infection d'origine dentaire de type cellulite génienne ou labiale; seul un interrogatoire bien mené et des examens complémentaires orientent le praticien vers le diagnostic de SMR [7, 9].

La prise en charge des patients atteints du SMR reste difficile. II n'existe pas de traitement de fond ou de traitement spécifique [1]. II est essentiellement symptomatique et dépend des répercussions esthétiques et des signes cliniques [2]. Le traitement de première intention est la clofazimine (Lamprène ${ }^{\circledR}$ ) à la dose de 100 mg/jour, pendant plusieurs mois (2 à 12 mois). II permet le plus souvent de prévenir les poussées d'œdème et d'obtenir une réduction de son volume [1, 2]. Au stade de fibrose fixée, et en l'absence de poussées évolutives, une correction chirurgicale des lèvres peut être tentée [1]. Pour la macrochéilie, la réhabilitation esthétique consiste à effectuer la résection de l'excédent labial par voie endobuccale $[8,17]$. Cette chirurgie qui ne laisse aucune cicatrice visible, comporte également une résection des glandes accessoires hypertrophiques [1, 8].
Quelques résultats encourageants ont été obtenus avec la thalidomide ${ }^{[18]}$. En cas de paralysie faciale, la corticothérapie générale ou intralésionnelle est le traitement de choix [19]. Une neurolyse ou une décompression nerveuse chirurgicale peut être proposée en l'absence d'évolution favorable en 1 ou 2 mois [17, 20,21]. Tous les foyers infectieux dentaires et parodontaux doivent être éliminés car ils pourraient entretenir la réponse immunologique et être à l'origine de la persistance des symptômes buccofaciaux $[2,21]$.

\section{CONCLUSION}

Le SMR est une affection rare, caractérisée par l'association de manifestations neurologiques (paralysie faciale) et buccales (œdème labial et langue plicaturée).

Bien que l'étiologie et la pathogénie du SMR restent imprécises, le traitement de l'œdème buccofacial et de la paralysie faciale comporte de nombreuses propositions thérapeutiques qui ne répondent que partiellement à l'attente des patients. De plus, ces traitements n'empêchent pas les récidives.

Le rôle de l'odontologiste est triple :

- Participer à l'établissement du diagnostic en cas de suspicion par un suivi régulier et attentif ; l'interrogatoire et l'examen clinique jouent un rôle capital, la biopsie labiale est indispensable.

- Lorsque le diagnostic est confirmé, adopter une attitude prophylactique :

- instaurer une hygiène buccodentaire rigoureuse et des visites régulières,

- procéder à l'élimination des foyers infectieux d'origine dentaire sous antibioprophylaxie.

- Accompagner psychologiquement le patient dans sa maladie et, le cas échéant, savoir l'orienter vers un chirurgien plasticien ou un neurochirurgien. médecine

buccale

chirurgie

buccale

VOL. $13, \mathrm{~N}^{\circ} 3$ 2007

page 149 
Tableau 1 : Signes majeurs et mineurs pour le diagnostic SMR [5]. Major and minor manifestations of the Melkersson-Rosenthal syndrome.

\begin{tabular}{|c|c|c|c|}
\hline $\begin{array}{l}\text { Signes } \\
\text { de } 1^{\text {er }} \text { ordre }\end{array}$ & $\begin{array}{c}\text { Signes } \\
\text { de } 2^{\mathrm{e}} \text { ordre }\end{array}$ & $\begin{array}{c}\text { Signes } \\
\text { de } 3^{e} \text { ordre }\end{array}$ & Diagnostic \\
\hline $\begin{array}{l}\text { Paralysie faciale + } \\
\text { œdème labial ou facial } \\
\text { d'aspect histologique } \\
\text { caractéristique } \\
\text { Lésions œdémateuses } \\
\text { d'aspect histologique } \\
\text { caractéristique }\end{array}$ & $\begin{array}{l}\text { Avec ou sans } \\
\text { langue plicaturée }\end{array}$ & 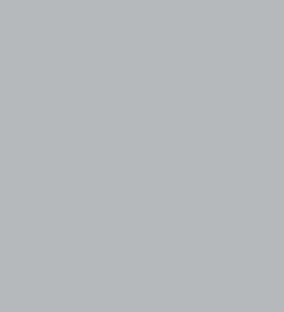 & SMR incomplet mais sûr \\
\hline Paralysie faciale & $\begin{array}{l}\text { CEdème d'aspect } \\
\text { histologique non } \\
\text { caractéristique } \\
\text { CEdème d'aspect } \\
\text { histologique non } \\
\text { caractéristique + } \\
\text { langue plicaturée } \\
\text { CEdème d'aspect } \\
\text { histologique non } \\
\text { caractéristique + } \\
2 \text { signes de } 2^{\circ} \text { ordre } \\
\text { CEdème d'aspect } \\
\text { histologique non } \\
\text { caractéristique } \\
+1 \text { signe de } 2^{\circledR} \text { ordre } \\
\text { Lésions d'aspect } \\
\text { histologique non } \\
\text { caractéristique } \\
\text { Lésions d'aspect } \\
\text { histologique non } \\
\text { caractéristique }\end{array}$ & $\begin{array}{l}3 \text { signes de } \\
3^{e} \text { ordre }\end{array}$ & $\begin{array}{l}\text { SMR incomplet mais sûr } \\
\text { SMR incomplet mais } \\
\text { probable } \\
\text { SMR incomplet mais } \\
\text { probable } \\
\text { SMR incomplet et } \\
\text { douteux } \\
\text { SMR incomplet et } \\
\text { douteux }\end{array}$ \\
\hline
\end{tabular}

Signes de $1^{\text {er }}$ ordre : paralysie faciale, œdème d'aspect histologique caractéristique.

Signes de $2^{\circ}$ ordre : langue plicaturée, œdème d'aspect histologique non caractéristique, céphalées à type de migraines, atteinte d'autres nerfs crâniens.

Signes de $3^{\circledR}$ ordre : symptômes végétatifs buccofaciaux, symptômes psychiatriques. 


\section{RÉFÉRENCES}

1 - Benyahya I, Mbirik K, El Kabli H. Syndrome de Melkersson-Rosenthal : à propos d'un cas. Actual Odonto-Stomatol 2004 ; 227 : 217-24.

2 - Cherkaoui A, Sahnoun I, Benrachadi L, Benzarti N. Le syndrome de Melkersson-Rosenthal : aspects buccodentaires. Espérance Médicale. Spéciale Dentaire 2004 ; $52: 21-4$.

3 - Grenne RM, Rogers RS III. Melkersson-Rosenthal Syndrome: a review of 36 patients. J Am Acad dermatol $1989 ; 21: 1263-70$.

4 - Stosiek N, Birolleau S, Capesius C, Hornstein OP. Chronicité et incertitudes diagnostiques du syndrome de Melkersson-Rosenthal. Ann Dermatol Venerol 1992 ; $119: 635-8$.

5 - Meisel-Stosiek M, Hornstein OP. Family study on Melkersson-Rosenthal syndrome. Acta Dermatol Venerol $1990 ; 70: 221-6$.

6 - Mainetti C, Masouye I. CEdème facial solide persistant du sujet jeune. Ann Dermatol Venerol 1994 ; 2 : 165 70.

7 - Benzarti M, Karfi M, Fazaa B, Zermani R, Zeglaoui F, Kanoun MR. Le syndrome de Melkersson-Rosenthal, aspects cliniques et évolutifs. Nouv Dermatol 1997 ; $16: 378-80$.

8 - Camacho F, Garcia-Bravo B, Carrizosa A. Treatment of Miescher's cheilitis granulomatosa in MelkerssonRosenthal syndrome. J Eur Acad Dermatol Venerol 2001; 15 : 546-9.

9 - Ang KL, Jones S. Melkersson-Rosenthal syndrome. J Laryngol Otol 2002 ; 116 : 386-8.

10 - Ziemmer WM, Rogers RS, Reeve CM, Sheridan PJ. Orofacial manifestations of Melkersson-Rosenthal syndrome. A study of 42 patients and review of 220 cases from the literature. Oral Surg Oral Med Oral Pathol $1992 ; 74: 610-9$.
11 - Rogers RS III. Granulomatous cheilitis, MelkerssonRosenthal syndrome, and orofacial granulomatosis. Arch Dermatol 2000 ; 136 : 1557-8.

12 - Kharoubi S. Melkersson-Rosenthal syndrome: clinical features and a suggested diagnostic grading system. Rev Laryngol Oto Rhinol (Bord) 1998 ; 119 : 109-13.

13 - Sciubba JJ, Said-Al-Naief N. Orofacial granulomatosis: presentation, pathology and management of 13 cases. J Oral Pathol Med 2003 ; 32 : 576-85.

14 - Bogenrieder T, Rogler G. Orofacial granulomatosis as the initial presentation of Crohn's disease in a adolescent. Dermatology 2003 ; 206 : 273-8.

15 - Cleary KR, Batsakis JG. Orofacial granulomatosis and Crohn's disease. Otol Rhinol Laryngol 1996 ; 105: 1667.

16 - Tatnall FM, Dodd HJ, Sarkany I. Crohn's disease with metastatic cutaneous involvement and granulomatous cheilitis. J R Soc Med 1987 ; 80 : 49-50.

17 - Ziem PE, Pfrommer C, Goerdt S, Orfanos CE, BlumePeytavi U. Melkersson-Rosenthal syndrome in childhood : a challenge in differential diagnosis and treatment. Br J Dermatol 2000 ; 143 : 860-3.

18 - Safa G, Joly P, Boulli MC, Lauret PH. Syndrome de Melkersson-Rosenthal traité par thalidomide. Ann Dermatol Venereol 1995 ; 122: 606-11.

19 - Ferriby D, Pertuzon B, Clarisse J, Vermesch P. Imagerie par résonance magnétique du nerf facial dans un cas de syndrome de Melkersson-Rosenthal. Rev Neurol $1998 ; 154: 426-8$.

20 - Dutt SN, Mirza S, Irving RM, Donaldson I. Total decompression of facial nerve for Melkersson-Rosenthal syndrome. J Laryngol Otol 2000 ; 114 : 870-3.

21 - Le Toux G. Syndrome de Melkersson-Rosenthal. Ann Oto-Laryngol 1989 ; 106 : 44-6.

\begin{tabular}{l}
$\begin{array}{l}\text { médecine } \\
\text { buccale } \\
\text { chirurgie } \\
\text { buccale }\end{array}$ \\
\hline VOL. 13, N ${ }^{\circ} 3$ \\
2007 \\
\hline page 151
\end{tabular}

page 151 